\title{
Balão Único versus Balão de Inoue na Valvoplastia Mitral Percutânea por Balão. Resultados Imediatos e Complicações
}

\author{
Edison C. Sandoval Peixoto, Paulo Sérgio de Oliveira, Mário Salles Netto, Ivana Picone Borges, \\ Ronaldo A. Villela, Pierre Labrunie, Cláudia Brum, Rodrigo T. Sandoval Peixoto, Marcello A. Sena, \\ Marta Labrunie, Ricardo T. Sandoval Peixoto, Daniela M. Burello
}

\author{
Rio de Janeiro - Niterói, RJ
}

\begin{abstract}
Objetivo - Avaliar os resultados imediatos e complicações da valvoplastia mitral percutânea por balão (VMPB), com o balão de Inoue (BI) e com o balão único $(B U)$.

Métodos - Dentre 390 procedimentos utilizaram-seo BI em 29 procedimentos e o BU de baixo perfil em 337. Não houve diferença na idade e sexo nos 2 grupos. O grupo BI era menos sintomático $(p=0,0015)$. Não houve diferença na distribuição do escore ecocardiográfico e da área valvar mitral (AVM) pré-VMPB.

Resultados - Quando compararam-se os 2 grupos entre si, os resultados nos grupos BI e BUforam, respectivamente: pré-VMPB para pressão pulmonar média (PPM) 36 \pm 15 e 39 $\pm 14 \mathrm{mmHg}, p=0,2033$, para gradiente

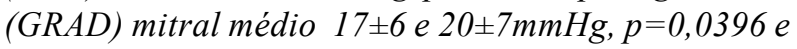
$A V M 0,9 \pm 0,2$ e 0,9 $99,2 \mathrm{~cm}^{2}, p=0,8043$, enquanto os valores pós-VMPB foram PPM $25 \pm 8$ e $28 \pm 10 \mathrm{mmHg}$, $p=0,2881, G R A D 5 \pm 3$ e $5 \pm 4 m m H g, p=0,2778$ e AVM $2,2 \pm 0,2$ e 2,0 $00,4 \mathrm{~cm}^{2}, p=0,0362$. Pré-VMPB a válvula mitral era competente em 26 procedimentos com o BI e 280 dos com o BU e havia regurgitação mitral de $+/ 4 \mathrm{em}$ 3 do grupo BI e em 57 do BU, $p=0,3591$ e pós-VMPB tivemos, no grupo BI a valva mitral (VM) competente em 18 , +/4 em 7 e 2+/4 em 4 e no grupo BU, a VMera competente em 218, +/4 em 80, 2+/4 em 25, 3+/4 em 5 e 4+/4 em 2, $p=0,7439$. Só houve complicações no grupo $B U$.

Conclusão - As duas técnicas foram eficientes. Os resultados hemodinâmicos foram semelhantes, embora a AVM pós-VMPB do grupo do BIfoi maior.
\end{abstract}

Palavras-chave: valvoplastia mitral percutânea por balão, balão de Inoue, balão único

\author{
Single Balloon versus Inoue Balloon in \\ Percutaneous Mitral Balloon Valvuloplasty. \\ Short-term Results and Complications
}

Purpose - To assess short-term results and complications of percutaneous mitral balloon valvuloplasty $(P M B V)$ performed with Inoue balloon (IB) and single low profile balloon $(S B)$.

Methods - We performed 390 PMBV procedures, 29 with $I B$ and 337 with $S B$. There were no differences in age, sex, echocardiographic score distribution and echocardiographic mitral valve area (MVA).

Results - We performed 29 complete procedures with $I B$ and 330 of 337 in SB group. Comparing IB and pre and pos-PMBV data we obtained: mean pulmonary artery pressure (MPAP) $36 \pm 15$ and $39 \pm 14 \mathrm{mmHg}, p=0.2033$, mean mitral gradient $17 \pm 6$ and $20 \pm 7 \mathrm{mmHg}, p=0.0396$ and $M V A$ $0.9 \pm 0.2$ and $0.9 \pm 0.2 \mathrm{~cm}^{2}, p=0.8043$ and pos $-P M B V: M P A P$ $25 \pm 8$ and $28 \pm 10 \mathrm{mmHg}, p=0.2881$, gradient $5 \pm 3$ and $5 \pm 4 \mathrm{mmHg}, p=0.2778$ and $M V A 2.2 \pm 0.2$ and $2.0 \pm 0.4 \mathrm{~cm}^{2}$, $p=0.0362$. Mitral valve (MV) was competent in 26 patients in $I B$ and in 280 in SB group and we had +/4 mitral regurgitation in 3 patients in $I B$ and in 57 in $S B$ group $(p=0.3591)$ pre- $P M B V$ respectively and pos- $P M B V$ there was also no difference in $M V$ competence $(p=0.7439)$.

Conclusion - Both techniques were effective. Hemodynamic data were also similar although $M V A$ was greater in IB group after $P M B V$.

Key-words: percutaneous mitral balloon valvuloplasty, Inoue balloon technique, single balloon technique

Arq Bras Cardiol, volume $71\left(n^{\circ} 1\right), 59-64,1998$

Cinecor (Pró-Cardíaco e $4^{\circ}$ Centenário), Rio de Janeiro e Hospital Universitário Antônio Pedro da UFF, Niterói

Correspondência: Edison C. Sandoval Peixoto - Av. Epitácio Pessoa, 4986/301 - 22471-001 - Rio de Janeiro, RJ

Recebido para publicação em 12/2/98

Aceito em 13/5/98
Em 1984, Inoue e col ${ }^{1}$ publicaram a técnica de dilatação da valva mitral (VM) percutânea por balão, utilizando o balão que leva seu nome. Em seguida, Lock e col${ }^{2}$ aplicaram a técnica a crianças. Reifart e $\mathrm{col}^{3}$ mostraram a possibilidade de dilatar a VM calcificada através de estudo experimental, Mckay e col ${ }^{4}$ e Palacios e col ${ }^{5}$ colocaram-na em prática nos 
Estados Unidos. Na Arábia Saudita, Al Zaibag e col ${ }^{6}$ passaram a usar a técnica do duplo balão por via transeptal, para obtenção de maior área valvar mitral após o procedimento.

Babic e $\operatorname{col}^{7}$ descreveram outra técnica de dilatação da VM, onde o fio guia e o cateter balão são introduzidos, retrogradamente, pela aorta. Entre nós, Mossmann e col ${ }^{8}$ e Buchler e $\operatorname{col}^{9}$ descreveram uma nova técnica por via retrógrada.

Iniciamos a valvoplastia mitral transeptal por balão (VMPB) em 1987, utilizando um único balão ${ }^{10}$ e, logo a seguir, a técnica do duplo balão ${ }^{11,12}$. Continuamos a publicar nossa experiência com a VMPB ${ }^{13,14}$ e viemos a utilizar a técnica do balão de Inoue (BI) ${ }^{15}$ e do balão único de baixo perfil (BU) ${ }^{16}$. Essas duas últimas técnicas são as de maior número de casos na nossa experiência e temos obtidos bons resultados e baixo nível de complicações ${ }^{17-20}$.

A técnica da valvoplastia por via transarterial ficou mais ou menos restrita aos relatos iniciais ${ }^{7-9}$, enquanto a VMPB passou a ser a $1^{\mathrm{a}}$ escolha de tratamento para estenose mitral (EM) grave, sintomática, que não apresente grande acometimento do aparelho subvalvar mitral ou grande calcificação e espessamento dos folhetos da VM. Demonstrou persistência dos resultados obtidos ${ }^{21}$, passando a anteceder a comissurotomia mitral cirúrgica nesses pacientes.

No presente trabalho, comparamos os resultados e complicações das técnicas do BI e do BU na VMPB, predominantes na nossa experiência ${ }^{22}$, e duas das três técnicas em uso no nosso meio, atualmente, já que a técnica do duplo balão também é utilizada. Na literatura há trabalhos de comparação do BI com o duplo balão e do duplo balão com o BU convencional ${ }^{23-25}$, como o que utilizamos no início da nossa experiência ${ }^{10}$ mas não há comparações com o BU de grande diâmetro e baixo perfil a não ser as nossas ${ }^{22}$.

\section{Métodos}

Dentre 390 procedimentos de VMPB realizados entre 6/7/87 a 28/2/97, foram estudados, prospectivamente, os resultados obtidos em 366 procedimentos, sendo 337 com o BUe 29 comoBI.

Dos 29 procedimentos com o BI, $23(79,3 \%)$ pacientes eram do sexo feminino e $6(20,7 \%)$ do sexo masculino, enquanto no grupo do BU $275(81,6 \%)$ eram mulheres e 62 $(18,4 \%)$ homens, $\mathrm{p}=0,7607$, não sendo a diferença significativa. A idade do grupo do BI foi $38 \pm 10$ anos e a do BU $37 \pm 13$ anos, $\mathrm{p}=0,7333$.

No grupo do BI, a classe funcional (CF) da NYHA era de grau Iem 2(6,9\%) procedimentos, CFII em 10(34,5\%), CFIII em $14(48,3 \%)$ e CFIV em $3(10,3 \%)$, enquanto no grupo doBU a CF foi I em 2(0,6\%) procedimentos, CFII em 61 (18,1\%), CF III em 239(70,9\%)eCFIV em 35 (10,4\%) p=0,0018.

No grupo do BI em três procedimentos os pacientes já haviam sido submetidos a comissurotomia cirúrgica prévia, enquanto no grupo do BU, em 31 procedimentos, tinha havido comissurotomia cirúrgica prévia, $\mathrm{p}=0,8383$. VMPB prévia só havia ocorrido no grupo do BU (6 procedimentos).

A área valvar mitral (AVM) determinada por ecocardiografia pelo halfpressure time foi de $1,0 \pm 0,2 \mathrm{~cm}^{2}$, no grupo do BI, e de $0,9 \pm 0,2 \mathrm{~cm}^{2}$, no BU, $\mathrm{p}=0,5998$ e o escore ecocardiográfico foi, no grupo do BI, 5 em 2 procedimentos, 6 em 5 , 7 em 6, 8 em 12, 9 em 3 e 10 em 1 e, no grupo do BU, o escore foi $4 \mathrm{em} 10$ procedimentos, $5 \mathrm{em} 23,6 \mathrm{em} 75,7 \mathrm{em} 65,8 \mathrm{em} \mathrm{123,}$ 9 em 23, 10 em 13, 11 em 3, 12 em 1 e 14 em 1,p=0,9863.

Em todos os procedimentos realizou-se primeiramente o cateterismo direito e esquerdo através da veia e artéria femorais esquerdas. A dilatação septal foi feita com balões de 6, 7 ou 8mm na técnica do BU e com dilatador 14 F na técnica do BI. Na técnica do BI utilizaram-se diâmetros máximos de insuflação, variando de 24 a $28 \mathrm{~mm}$. Na técnica do BU utilizaram-se balões de $25 \mathrm{~mm}$ ou $30 \mathrm{~mm}$ de diâmetro ou ainda balão de 25 seguido de balão de $30 \mathrm{~mm}$. Após a dilatação mitral foi sempre realizada uma ventriculografia esquerda em OAD, além de novo cateterismo direito e esquerdo e nova medida do gradiente (GRAD) entre átrio esquerdo (AE) e ventrículo esquerdo (VE). Foi sempre realizada a medida do GRAD entre AE e VE simultâneo, imediatamente, antes e após a dilatação da VM. Foram medidos GRADs protodiastólico, mesodiastólico e telediastólico e o GRAD médio pelo método dos três pontos, como a média aritmética das três medidas anteriores ${ }^{26}$. Foi determinada a área valvar mitral (AVM) pré e pós-dilatação. Determinou-se o débito cardíaco por termodiluição e, utilizando-se, a seguir, a fórmula de Gorlin e Gorlin ${ }^{27}$ para o cálculo da área. A presença de insuficiência mitral (IM) foi graduada segundo o critério semi-quantitativo de Sellers e $\mathrm{col}^{28}$.

Foram estudados idade, sexo, CF da NYHA pré procedimento, VMPB ou comissurotomia cirúrgica prévia, escore ecocardiográfico da VM, área ecocardiográfica da VM pré, pressão pulmonar média (PPM) pré e pós-VMPB, GRAD médio entre AE e VE pré e pós-VMPB, área valvar hemodinâmica da VM pré e pós-VMPB, regurgitação mitral (RM) pré e pós-VMPB, complicações e mortalidade. Compararamse os dados obtidos nos dois grupos.

A análise estatística foi realizada com a utilização do programa EPI-INFO ${ }^{29}$, tendo-se determinado a freqüência das variáveis qualitativas e numéricas. As variáveis numéricas foram também estudadas através da análise da variância e as qualitativas pelo teste do qui-quadrado.

\section{Resultados}

Foram realizados 366 procedimentos, sendo 29 com BI e 337 com BU, entretanto, dos 337 procedimentos com BU apenas 330 foram completos com dados pré e pós-VMPB pois, sete não foram efetivados (colocação do balão na válvula mitral com sua insuflação).

No grupo do BI, a PPM pré-VMPB foi de $36 \pm 15 \mathrm{mmHg}$ e pós-VMPB de $25 \pm 8 \mathrm{mmHg}(\mathrm{p}=0,0058)$, o GRAD foi $17 \pm 6 \mathrm{e}$ $5 \pm 3 \mathrm{mmHg}(\mathrm{p}<0,000001)$ e a AVM ${ }^{27}$ de $0,9 \pm 0,2$ e $2,2 \pm 0,2 \mathrm{~cm}^{2}$ $(\mathrm{p}<0,000001)$, respectivamente (tab. I).

No grupo do BU, a PPM pré-VMPB foi de $39 \pm 14 \mathrm{mmHg}$ e pós-VMPB de $27 \pm 11 \mathrm{mmHg}$ (p<0,000001), o GRAD foi $20 \pm 7$ e $5 \pm 4 \mathrm{mmHg}(\mathrm{p}<0,000001)$ e a $\mathrm{AVM}^{27}$ de $0,9 \pm 0,2$ e $2,0 \pm 0,4 \mathrm{~cm}^{2}$ $(\mathrm{p}<0,000001)$, respectivamente (tab. II). 


\begin{tabular}{|c|c|c|c|}
\hline \multicolumn{4}{|c|}{$\begin{array}{c}\text { Tabela I - Resultados hemodinâmicos imediatos da VMPB no } \\
\text { grupo do balão de Inoue }(n=29)\end{array}$} \\
\hline & Pré-VMPB & Pós-VMPB & $\mathrm{p}$ \\
\hline $\begin{array}{l}\text { Pressão pulmonar média } \\
(\mathrm{mmHg})\end{array}$ & $36 \pm 15$ & $25 \pm 8$ & 0,0058 \\
\hline $\begin{array}{l}\text { Gradiente mitral médio } \\
(\mathrm{mmHg})\end{array}$ & $17 \pm 6$ & $5 \pm 3$ & $<0,000001$ \\
\hline Área valvar mitral $\left(\mathrm{cm}^{2}\right)$ & $0,9 \pm 0,2$ & $2,2 \pm 0,2$ & $<0,000001$ \\
\hline
\end{tabular}

\begin{tabular}{|c|c|c|c|}
\hline \multicolumn{4}{|c|}{$\begin{array}{l}\text { Tabela II - Resultados hemodinâmicos imediatos da VMPB no } \\
\text { grupo do balão único }(\mathrm{n}=\mathbf{3 3 0})\end{array}$} \\
\hline & Pré-VMPB & Pós-VMPB & $\mathrm{p}$ \\
\hline $\begin{array}{l}\text { Pressão pulmonar média } \\
(\mathrm{mmHg})\end{array}$ & $39 \pm 14$ & $27 \pm 11$ & $<0,000001$ \\
\hline $\begin{array}{l}\text { Gradiente mitral médio } \\
(\mathrm{mmHg})\end{array}$ & $20 \pm 7$ & $5 \pm 4$ & $<0,000001$ \\
\hline Área valvar mitral $\left(\mathrm{cm}^{2}\right)$ & $0,9 \pm 0,2$ & $2,0 \pm 0,4$ & $<0,000001$ \\
\hline
\end{tabular}

\begin{tabular}{|c|c|c|c|}
\hline \multicolumn{4}{|c|}{$\begin{array}{l}\text { Tabela III - Comparação dos valores hemodinâmicos pré-VMPB } \\
\text { nos } 2 \text { grupos }\end{array}$} \\
\hline & $\begin{array}{l}\text { Balão de Inoue } \\
\qquad(\mathrm{n}=29)\end{array}$ & $\begin{array}{l}\text { Balão único } \\
\qquad(\mathrm{n}=330)\end{array}$ & $\mathrm{p}$ \\
\hline $\begin{array}{l}\text { Pressão pulmonar média } \\
(\mathrm{mmHg})\end{array}$ & $36 \pm 15$ & $39 \pm 14$ & 0,2033 \\
\hline $\begin{array}{l}\text { Gradiente mitral médio } \\
(\mathrm{mmHg})\end{array}$ & $17 \pm 6$ & $20 \pm 7$ & 0,0396 \\
\hline Área valvar mitral $\left(\mathrm{cm}^{2}\right)$ & $0,9 \pm 0,2$ & $0,9 \pm 0,2$ & 0,8043 \\
\hline
\end{tabular}

Quando compararam-se os dados hemodinâmicos dos dois grupos entre si pré-VMPB, encontramos nos grupos do BI e do BU, respectivamente: $P P M 36 \pm 15$ e $39 \pm 14 \mathrm{mmHg}$ $(\mathrm{p}=0,2033)$, GRAD $17 \pm 6 \mathrm{e} 20 \pm 7 \mathrm{mmHg}(\mathrm{p}=0,0396) \mathrm{e} \mathrm{AVM}^{27}$ $0,9 \pm 0,2 \mathrm{e} 0,9 \pm 0,2 \mathrm{~cm}^{2} \mathrm{p}=0,8043$ (tab. III).

Quando compararam-se os dados hemodinâmicos dos dois grupos entre si pós-VMPB, encontramos nos grupos do BI e do BU, respectivamente: PPM $25 \pm 8$ e $28 \pm 10 \mathrm{mmHg}$ $(\mathrm{p}=0,2881)$, GRAD $5 \pm 3$ e $5 \pm 4 \mathrm{mmHg}(\mathrm{p}=0,2778)$ e $\mathrm{AVM}^{27}$ $2,2 \pm 0,2 \mathrm{e} 2,0 \pm 0,4 \mathrm{~cm}^{2}(\mathrm{p}=0,0362)$ (tab.IV).

No estudo hemodinâmico pré-VMPB, a competência da VM ou a presença de $\mathrm{RM}^{28}$ no grupo do BI foi $0+$ em 26 procedimentos e $1+\mathrm{em} 3$ e no grupo do BU foi $0+\mathrm{em} 280 \mathrm{e} 1+$ 57 procedimentos $(\mathrm{p}=0,3591)$ (tab. V). Pós-VMPB, a competência da $\mathrm{VM}$ ou a presença de $\mathrm{RM}^{28}$ no grupo do $\mathrm{BI}$ foi $0+$ em 18 procedimentos, $1+\mathrm{em} 7 \mathrm{e} 2+\mathrm{em} 4 \mathrm{e}$ no grupo do $\mathrm{BU}$ foi $0+$ em $218,1+\mathrm{em} 80,2+\mathrm{em} 25,3+\mathrm{em} 5 \mathrm{e} 4+\mathrm{em} 2$ procedimentos ( $\mathrm{p}=0,7439)$ (tab. VI).

Só ocorreram complicações no grupo do BU. Além dos sete pacientes com IM grave ( $3 \mathrm{e} 4+$ ) 1,9\% do total de procedimentos efetivados e $2,1 \%$ dos mesmos realizados com o BU. Ocorreram um episódio de acidente vascular cerebral (AVC) equivalente a $0,3 \%$ dos pacientes submetidos a VMPB e quatro de tamponamento cardíaco (dois por perfuração de VE seguidas de cirurgia de emergência e óbito, um

\begin{tabular}{|c|c|c|c|}
\hline \multicolumn{4}{|c|}{$\begin{array}{l}\text { Tabela IV - Comparação dos valores hemodinâmicos pós-VMPB } \\
\qquad \text { nos } 2 \text { grupos }\end{array}$} \\
\hline & $\begin{array}{l}\text { Balão de Inoue } \\
\quad(\mathrm{n}=29)\end{array}$ & $\begin{array}{l}\text { Balão único } \\
(\mathrm{n}=330)\end{array}$ & $\mathrm{p}$ \\
\hline $\begin{array}{l}\text { Pressão pulmonar média } \\
(\mathrm{mmHg})\end{array}$ & $25 \pm 8$ & $28 \pm 10$ & 0,2881 \\
\hline $\begin{array}{l}\text { Gradiente mitral médio } \\
(\mathrm{mmHg})\end{array}$ & $5 \pm 3$ & $5 \pm 4$ & 0,2778 \\
\hline Área valvar mitral $\left(\mathrm{cm}^{2}\right)$ & $2,2 \pm 0,2$ & $2,0 \pm 0,4$ & 0,0362 \\
\hline
\end{tabular}

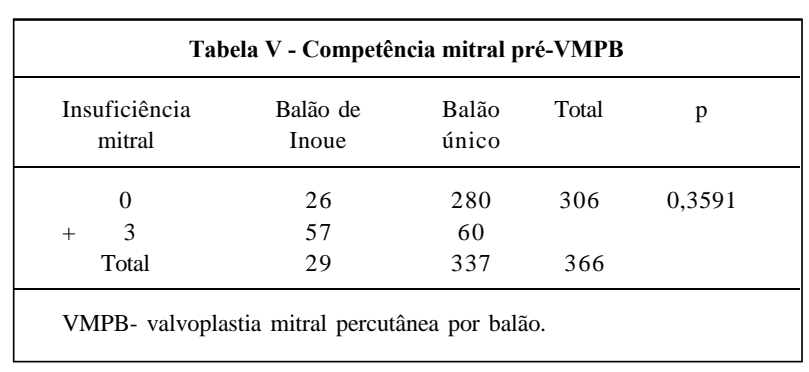

\begin{tabular}{|ccccc|}
\hline \multicolumn{5}{|c|}{ Tabela VI - Competência mitral pós-VMPB } \\
\hline $\begin{array}{c}\text { Insuficiência } \\
\text { mitral }\end{array}$ & $\begin{array}{c}\text { Balão de } \\
\text { Inoue }\end{array}$ & $\begin{array}{c}\text { Balão } \\
\text { único }\end{array}$ & Total & $\mathrm{p}$ \\
\hline 0 & 18 & 218 & 236 & \\
+ & 7 & 80 & 87 & \\
++ & 4 & 25 & 29 & 0,7439 \\
+++ & 0 & 5 & 5 & \\
++++ & 0 & 2 & 2 & \\
Total & 29 & 330 & 359 & \\
\hline
\end{tabular}

por perfuração de $\mathrm{AE}$ seguida de cirurgia de emergência e recuperação do paciente e um controlado por drenagem pericárdica e boa evolução). Os quatro episódios de tamponamento cardíaco representaram $1,1 \%$ dos procedimentos e $1,2 \%$ dos procedimentos com o BU. Com já referido tivemos dois óbitos ( $0,5 \%$ do total de procedimentos e $0,6 \%$ dos com o BU) secundários a tamponamento cardíaco por perfuração de VE.

\section{Discussão}

Introduzida por Inoue ${ }^{1}$ a VMPB firmou-se rapidamente com um dos tratamentos da EM grave. Evidenciou-se de início que a área mitral pós-VMPB era maior quando utilizava-se a técnica do duplo balão, em vez da do BU convencional ${ }^{6,23,30}$. A seguir, mostrou-se que com balão bifoil e trifoil obtinha-se também AVM pós-VMPB semelhante à obtida com a técnica do duplo balão ${ }^{24}$. Foram demonstrados, também, a eficiência e baixo nível de complicações com o BI ${ }^{31,32}$. Evidenciou-se ainda que os valores obtidos com o duplo balão ${ }^{33} \mathrm{e}$ os resultados imediatos com o duplo balão e com o BI eram semelhantes ${ }^{34}$.

Hoje está comprovado que se pode obter AVM pósVMPB semelhante com qualquer das técnicas em uso, quer seja do duplo balão, do BI ou do balão monofoil de baixo 
perfil (BU), desde que as áreas efetivas de dilatação dos balões sejam comparáveis ${ }^{16,19,22,25}$.

A técnica de VMPB via arterial retrógrada ficou restrita aos relatos iniciais e seria de uso excepcional, como alternativa, a via transeptal ${ }^{8,9,35}$.

Comparamos os resultados que obtivemos com a técnica do BI e do BU, que foram os mais utilizados na nossa experiência, sendo 337 procedimentos com o BU e 29 com o BI. Quando compararam-se idade e sexo dos dois grupos não houve diferença significativa, entretanto, o grupo do BU apresentava maior percentual de pacientes em CF III IV IV com diferença significativa, portanto, clinicamente mais grave. $\mathrm{Na}$ comparação dos dados hemodinâmicos pré-VMPB, PPMe AVM não apresentaram diferença significativa, entretanto, $o$ GRAD médio entre AEe VE foi maior no grupo do BU o que pôde evidenciar maior gravidade desse grupo.

Tanto o BI quanto o BU foram eficientes no tratamento da EM grave, diminuindo de forma significativa PPM e GRAD mitral médio e aumentando a AVM. Analisando-se os resultados hemodinâmicos pós-VMPB não se encontrou diferença significativa entre os dois grupos para PPM e GRAD mitral, entretanto, a AVM foi um pouco menor no grupo do BU, com diferença significativa. Esse achado evidenciou menor eficiência desse balão ou a maior gravidade desse grupo. O escore ecocardiográfico dos dois grupos, quando comparados entre si, não mostrou diferença significativa e, portanto, não poderia ser a causa desse achado.

Na maioria dos trabalhos iniciais, a AVM aumentava de menos de $1 \mathrm{~cm}^{2}$ para pelo menos $2 \mathrm{~cm}^{236-39} \mathrm{etrabalhos} \mathrm{posteri-}$ ores mostraram AVM após a VMPB um pouco $<2 \mathrm{~cm}^{2}$, independente do uso de técnica do duplo balão ou do balão de Inoue ${ }^{25,40,41}$. Já os trabalhos mais recentes têm mostrado AVM média $\geq 2 \mathrm{~cm}^{2}$ independente de ser a técnica utilizada do BI, do duplo balão ou do BU ${ }^{19,22,33}$. Nossos achados estão de acordo com o descrito na literatura. O mesmo ocorreu com a diminuição da PPM e do GRAD mitral médio pós-VMPB ${ }^{36}$.

A AVM após a VMPB está diretamente relacionada ao tamanho dos balões de valvoplastia. Os balões de valvotomia devem ser suficientemente grandes para proporcionar um bom resultado, mas não tão grandes a ponto de causar IM. A incidência de IMé menor quando se corrige a área efetiva da dilatação do balão para a área de superfície corporal, ficando $\leq 4,0 \mathrm{~cm}^{242}$. Usamos área efetiva de dilatação relativamente elevada ao longo da nossa experiência ${ }^{17-20,22}$, sem que houvesse aumento de RM, bem como no presente trabalho.

O registro de valvoplastia mitral por balão do NHLBI identificou maior área valvar com a técnica do duplo balão do que com a técnica do BU. Usou balões de acetileno, comentando que na técnica do BU não usou os balões maiores do tipo Inoue ${ }^{43}$. A incidência de IM foi semelhante nos dois grupos. Casele e $\mathrm{col}^{44}$, também, obtiveram bons resultados com a técnica do duplo balão. Salientamos que a literatura não evidencia diferença de resultados, quando se utiliza o BU ou o duplo balão se as áreas efetivas de dilatação forem similares $^{25}$. Tanto o BI quanto o BU de baixo perfil, que utilizamos, possibilitaram a utilização de áreas efetivas de dilatação similares às da técnicas de duplo balão com um único balão.
Apenas sete, todos no grupo do BU, dos 366 procedimentos não foram efetivados, ou seja, o balão foi colocado na VMe inflado, dando a impressão de ser essa técnica um pouco mais difícil de ser realizada que a do BI (na nossa opinião, em grau de dificuldade, a técnica do BU é intermediária entre a do BI, que é mais simples e a do duplo balão que é a de maior dificuldade, entretanto, quanto a custo, a do BU é a menos dispendiosa e a do BIé a de maior custo) ou a período diverso da experiência, quanto a utilização das duas técnicas.

Pré-VMPB havia apenas pacientes com VM competente ou RM 1+/4 e sem diferença significativa (tab. V). PósVMPB também não houve diferença significativa mas houve sete pacientes com IM grave, todos no grupo do BU (tab. VI), correspondendo a $1,9 \%$ do total dos pacientes com procedimentos efetivados e 2,1\% dos do grupo do BU.

$\mathrm{O}$ aparecimento de IM ou seu aumento é relatado na literatura, podendo chegar até a metade dos $\operatorname{casos}^{1,24,36-38,45}$, sendo em geral discreto. Relato de IM de significado ( 3 ou $4+)$ é menor ainda ${ }^{41,45-49}$. Casele e col $^{44}$ encontraram aumento de $1+\mathrm{em} 31 \%$ dos seus procedimentos, de $2+\mathrm{em} 13 \%$ e de $4+$ em apenas $0,9 \%$, superando nossa incidência de IM pósVMPB. Hernandez e $\mathrm{col}^{48}$ relataram IM grave (4+) em 6,6\% de seus procedimentos, achado muito superior ao nosso. No estudo de Aurora e col ${ }^{50}$ é concluído que o tamanho do balão, o grau de doença do aparelho subvalvar ou a gravidade da EM não têm relação com o aparecimento de IM. Já para Roch e col $^{51}$ só predisseram aumento da RM a relação da área efetiva de dilatação do balão com a superfície corporal. Apesar da elevada área efetiva de dilatação utilizada por nós, em ambos os grupos, a nossa incidência de IM de significado foi baixa.

Outras complicações também só ocorreram no grupo do BU. Tivemos 1 episódio de AVC correspondendo a 0,3\% dos pacientes submetidos a VMPB. Os episódios de embolização são raros na literatura, estando abaixo de 1 a $5 \%{ }^{2,52}$. O percentual de $0,3 \%$ de episódios embólicos na nossa casuística está abaixo do limite inferior da literatura.

Ainda ocorreram, como complicação grave, quatro episódios de tamponamento cardíaco $(1,1 \%$ do total dos 366 procedimentos realizados e $1,2 \%$ dos procedimentos com o BU), o que é um percentual baixo frente à literatura, que relata incidência de tamponamento cardíaco entre $0 \mathrm{e}$ $9 \%$ 1,37,39,50,52-55. O percentual de tamponamento cardíaco da nossa série foi baixo em relação a literatura.

Dos quatro casos de tamponamento cardíaco desta série, um foi contornado com drenagem pericárdica e ficou sem etiologia definida. Os outros três foram enviados à cirurgia cardíaca de emergência, tendo um ocorrido por perfuração de $\mathrm{AE}$, que foi suturada e teve evolução favorável, enquanto os dois restantes ocorreram por perfuração de VE e evoluíram para óbito apesar da cirurgia de emergência.

Foram $0,5 \%$ de óbitos do total dos 366 procedimentos realizados e $0,6 \%$ dos 337 procedimentos com o BU, o que é uma incidência baixa frente à literatura, que sugere que a mortalidade na VMPB seja de 1\%, apesar de haver registros de mortabilidade entre 0 e $6 \%{ }^{1,52,5456}$. Oóbito, na maioria das vezes, é devido à perfuração de VE ou IM aguda grave ${ }^{13,24,47,50}$.O 
NHLBI Balloon Valvuloplasty Registry ${ }^{57}$ relata num conjunto de 738 pacientes de vários centros, complicações graves em $12 \%$, com uma mortalidade no laboratório de oito $(1 \%)$ pacientes, ocorrendo óbito, relacionado ao procedimento, em $12(1,6 \%)$ pacientes.

Concluímos que ambas as técnicas foram eficientes no tratamento da EM grave, já que ambos os grupos tinham características clínicas semelhantes, embora a presença de menos sintomas e menor GRAD do BI mostre que o mesmo era menos grave, que a AVM obtida no grupo do BU, estatisticamente menor que a do grupo do BI, e essa diferença não deva ter significado clínico, e possa ter sido devida à diferença das técnicas ou à possibilidade do grupo do BI ser menos grave, que a incidência de IM, semelhante nos dois grupos, apesar de só ter havido IM grave no grupo do BU e, finalmente, que outras complicações maiores só ocorreram no grupo do BU, possivelmente devidas ao diverso período de experiência ou à técnica do BU, um pouco mais difícil que a do BI.

\section{Referências}

1. Inoue K, Owki T, Kikamara T, Kitamura F, Miyamoto M-Clinical application of ${ }^{\star}$ transvenous mitral comissurotomy by a new balloon catheter. J Thorac Cardiovasc Surg 1984; 87: 394-402.

2. Lock JE, Khalilulhah M, Shrivasta S, Bahl V, Klane JF - Percutaneous catheter comissurotomy in rheumatic mitral valve stenosis. N Engl J Med 1985; 313: 1515-18.

3. Reifart N, Nowark B, Baykut D, Bussman WD, Kalten-Bach M - Experimental mitral valvuloplasty of fibrotic and calcified valves with balloon catheters. J Am Coll Cardiol 1985; 5: 448.

4. McKay RG, Lock JE, Klane JF, Safian RD, Aroesty JM - Percutaneous mitral valvoplasty in an adult patient with calcific rheumatic mitral stenosis. J Am Coll Cardiol 1986; 7: 1410-15.

5. Palacios I, Lock JE, Klane JF, Block PC - Percutaneous transvenous balloon valvotomy in a patient with severe calcified mitral stenosis. J Am Coll Cardiol 1986; 7: 1416-19.

6. Al Zaibag M, Kasab JA, Ribeiro PA, Fagih MR - Percutaneous double balloon mitral valvotomy for rheumatic mitral valve stenosis. Lancet 1986; 1: 757-61.

7. Babic VV, Pejcic P, Djurisic Z, Vicinic M, Grukococ SM - Percutaneous transarterial balloon valvoplasty for mitral valve stenosis. Am J Cardiol 1986; 57: 1101-4.

8. Mossmann RA, Blancher C, Koehler N et al - Valvoplastia mitral com cateter balão. Experiência inicial com uma nova técnica. Arq Bras Cardiol 1987; 49: 333-7.

9. Buchler JR, Braga SLN, Assis SF et al - Valvoplastia por balão na estenose mitral reumática pela técnica transarterial. Arq Bras Cardiol 1987; 49 (supl I): 102.

10. Peixoto ECS - Valvoplastia mitral por via transeptal. Uma nova técnica de tratamento da estenose mitral. Ars Curandi Cardiologia 1987; 9: 9-10.

11. Peixoto ECS - Valvoplastia mitral com duplo balão. Um avanço nesse método de tratamento da estenose mitral. Ars Curandi Cardiologia 1988; 10: 3-4.

12. Peixoto ECS, Baptista EM, Vieira WJM, Morgado LC, Souza RJE, Lemos VLS Valvoplastia mitral por via transeptal. Um novo método de tratamento da estenose mitral severa. Ars Curandi Cardiologia 1988; 10: 51-62.

13. Peixoto ECS, Baptista EM, Vieira WJM et al - Valvoplastia mitral por via transeptal. Resultados e experiência do primeiro ano. Rev SOCERJ 1988; 1: 37-44.

14. Peixoto ECS, Baptista EM, Vieira WJM, Labrunie P, Morgado LC - Valvoplastia mitral por via transeptal como tratamento da estenose mitral grave. Bras Radiol 1989; 22: 151-5.

15. Oliveira PS, Peixoto ECS, Labrunie P et al - Valvoplastia mitral pela técnica de Inoue. Primeiros casos no Brasil. Arq Bras Cardiol 1990; 55(supl B): 202.

16. Peixoto ECS, Salles Netto M, Oliveira PS et al - Valvoplastia mitral por via transeptal. Evolução de 56 meses e apresentação de uma nova técnica (balão monofoil de baixo perfil e diâmetro de 25 ou 30mm). Arq Bras Cardiol 1992; 59(supl II): 215.

17. Peixoto ECS - Valvoplastia mitral percutânea por balão. Resultados imediatos e complicações. Tese para concurso para professor titular de Cardiologia. Niterói: UFF, 1993.

18. Peixoto ECS, Oliveira PS, Salles Netto M et al - Valvoplastia mitral percutânea por balão. Resultados imediatos, complicações e evolução hospitalar. Arq Bras Cardiol 1995; 64: 109-16.

19. Peixoto ECS, Oliveira PS, Salles Netto M et al - Valvoplastia mitral percutânea com a técnica do balão único. Resultados imediatos, complicações e evolução intra-hospitalar. Arq Bras Cardiol 1996; 66: 267-73.

20. Peixoto ECS, Oliveira PS, Salles Netto M et al - Valvuloplastia mitral percutánea con balón: resultados inmediatos, complicaciones y evolución hospitalaria. Rev Latinoamericana de Hemodinamia, Angiografia y Terapeutica por Cateterismo 1996; 2: 67-75.
21. Peixoto E, Borges I, Neves A et al - Clinical and echocardiographic long-term follow-up in mitral balloon valvuloplasty. Echocardiographic score influence. Am J Cardiol 1997; 80(suppl 7A): 735.

22. Peixoto E, Oliveira $\mathrm{P}$, Salles $\mathrm{M}$ et al - Inoue balloon versus monofoil balloon in mitral valvuloplasty. Results and complications. Am J Cardiol 1997; 80(suppl 7A): 735 .

23. Chen CR, Huang ZD, Lo ZX, Cheng TO - Comparision of single rubber nylon balloon and double polyethylene balloon valvoplasty in 94 patients with rheumatic mitral stenosis. Am Heart J 1990; 119: 102-11.

24. Patel J, Vylhilingum S, Mitha AS - Balloon dilatation of the mitral valve by a single, bifoil ( 2 x 19mm) or trifoil (3 x 15mm) catheter. Br Heart J 1990; 64: 342-6.

25. Ribeiro PA, Fawzy ME, Arafat MA et al - Comparison of mitral valve area results of balloon mitral valvotomy using the Inoue and double balloon techniques. Am J Cardiol 1991; 68: 687-8.

26. Yang SS, Bentivoglio L, Maranhão V, Golberg H - From cardiac catherization data to hemodynamic parameters. $2^{\text {nd }}$ ed. Philadelphia: FA Davis, 1978: 1-54.

27. Gorlin R, Gorlin SG - Hydraulic formula for calculation of the area of the stenotic mitral valve, other cardiac values and central circulatory shunts. Am Heart J 1951; 41: 1-29.

28. Sellers RD, Levy MJ, Amplatz K, Lillehei CW - Left retrogade cardioangiography in acquired cardiac disease. Technic, indication and interpretations in 700 cases. Am J Cardiol 1964; 14: 437-47.

29. Dean AG, Dean JA, Dicker RC - Epi-Info, version 5: a word processing, database and statistic program for epidemiology on mi-crocomputers. Stone Montain: UND, Incorporate, 1990.

30. Waller BF, Vantassel JW, McKay C - Anatomic basis for and morfologic results from catheter balloon valvoplasty of stenotic mitral valves. Clin Cardiol 1990; 13: 655-61.

31. Nishimura RA, Holmes Jr J, Rucler GS - Eficacy of percutaneous mitral balloon valvuloplasty with the Inoue balloon. Mayo Clin Proc 1991; 66: 276-82.

32. Feldman T, Carroll JD - Valve deformity and balloon mechanics in percutaneous transvenous mitral commissurotomy. Am Heart J 1991; 121: 1628-33.

33. Farhat MB, Belbout F, Gamra $\mathrm{H}$ et al - Results of percutaneous double-balloon mitral commissurotomy in one medical center in Tunisia. Am J Cardiol 1995; 76 : 1266-70.

34. Cardoso LF, Grinberg M, Patrício M et al - Estudo comparativo entre balão único de Inoue e duplo balão na valvoplastia mitral percutânea. Resultados imediatos e após seguimento de um ano. Arq Bras Cardiol 1996; 66: 213-6.

35. Stefanadis C, Kourovklis C, Stratos C, Pitsavos C, Tentolouris C, Toutouzas P Percutaneous balloon mitral valvoplasty by retrogade left atrial catheterization. Am J Cardiol 1990; 65: 650-4.

36. Palacios IF, Block PC, Wilkins GT, Weyman AE - Follow-up of patients undergoing mitral balloon valvotomy: Analysis of factors determining reestenosis. Circulation 1989; 79: 573-9.

37. Vahanian A, Michel PL, Cormier B et al - Results of percutaneous mitral commissurotomy in 200 patients. Am J Cardiol 1989; 63: 847-52.

38. Palacios I, Block PC, Brandi S et al - Percutaneous balloon valvotomy for patient with severe mitral stenosis. Circulation 1987; 75: 778-84.

39. Rocha P, Berland J, Mechmeche R et al - Valvuloplastia percutânea mitral por balão. Resultados imediatos de 80 casos. Arq Bras Cardiol 1989; 52: 253-8.

40. Ortiz AF, Macaya C, Alfonso F et al - Mono versus double-balloon technique for commissural splitting after percutaneous mitral valvotomy. Am J Cardiol 1992; 69: 1100-1.

41. Feldman T, Carrol JD, Isner JM et al - Effect of valve deformity on results and mitral regurgitation after Inoue balloon commissorotomy. Circulation 1992; 85: 180-7.

42. Abascal VW, Wilkins GT, Choong CY, Block PC, Palacios IF, Weyman AE Mitral regurgitation after percutaneous balloon mitral valvuloplasty in adults: 
Evaluation by pulsed doppler echocardiography. J Am Coll Cardiol 1988; 11: 257-63.

43. NHLBI Balloon Valvuloplasty Registry - Multicenter experience with balloon mitral commissurotomy. Circulation 1992; 85: 448-61.

44. Casele P, Block PC, O'Shea JP, Palacios IF - Atrial septal defect after percutaneous mitral balloon valvoplasty: Imediate results and follow-up. J Am Coll Cardiol 1990; 15: 1300-4.

45. Nobuyushi M, Hamasaki N, Kumura T et al - Indications, complications and shortterm clinical outcome of percutaneous transvenous mitral commissurotomy. Circulation 1989; 80:782-92.

46. Hung JS, Chern MS, Wu JJ et al - Short and long-term results of catheter balloon percutaneous transvenous mitral commissurotomy. Am J Cardiol 1991; 67 : 854-62.

47. Tuzcu EM, Block PC, Palacios IF - Comparison of early versus late experience with percutaneous mitral balloon valvoplasty. J Am Coll Cardiol 1991; 17: $1121-4$.

48. Hernandez R, Macaya C, Bañuelos C et al - Predictors, mechanisms and outcome of severe mitral regurgitation complicating percutaneous mitral valvotomy with the Inoue balloon. Am J Cardiol 1992; 70: 1169-74.

49. Inoue K, Hung JS - Percutaneous transvenous mitral commissurotomy (PTMC):
The far east experience. In: Topol EJ - Textbook of interventional cardiology. Philadelphia: WB Saunders, 1990: 887-99.

50. Aurora R, Nair M, Kaha GS et al - Non surgical mitral valvoplasty for rheumatic mitral stenosis. Indian Heart J 1990; 42: 329-34.

51. Roth RB, Block PC, Palacios IF - Predictor of increased mitral regurgitation after mitral balloon valvotomy. Cathet Cardiovas Diagm 1990; 20: 17-21.

52. Pan M, Medina A, Lezo JS et al - Cardiac tamponade complicating mitral balloon valvuloplasty. Am J Cardiol 1991; 68: 802-5.

53. Block PC - Early results of mitral balloon valvoplasty for mitral stenosis: Report from the NHLBI registry. Circulation 1988; 78(suppl 2): 489.

54. Herrmann HC, Kleaveland JP, Hill JA et al - The M-heart percutaneous balloon mitral valvoplasty registry: initial rsults and early follow-up the M-heart group. J Am Coll Cardiol 1990; 15: 1221-6.

55. Ruiz CE, Lan FYK - Percutaneous double balloon valvoplasty (PDBV) in 41 adults with mitral stenosis. Circulation 1987; 76(suppl 4): 76

56. Rediker DE, Block PC, Abascal VM, Palacios IF - Mitral balloon valvoplasty for mitral reestenosis after surgical commissurotomy. J Am Coll Cardiol 1988; 11 252-6.

57. NHLBI Balloon Vavuloplasty Registry - Complications and mortality of percutaneous balloon mitral commissurotomy. Circulation 1992; 85: 2014-24. 